2014

\title{
Alternative optical concept for electron cyclotron emission imaging
}

\author{
J. X. Liu \\ T. Milbourne \\ College of William and Mary \\ M. Bitter \\ L. Delgado-Aparicio
}

Follow this and additional works at: https://scholarworks.wm.edu/aspubs

\section{Recommended Citation}

Liu, J. X., Milbourne, T., Bitter, M., Delgado-Aparicio, L., Dominguez, A., Efthimion, P. C., ... \& Kasparek, W. (2014). Alternative optical concept for electron cyclotron emission imaging. Review of Scientific Instruments, 85(11), 11D802.

This Article is brought to you for free and open access by the Arts and Sciences at W\&M ScholarWorks. It has been accepted for inclusion in Arts \& Sciences Articles by an authorized administrator of W\&M ScholarWorks. For more information, please contact scholarworks@wm.edu. 


\title{
Alternative optical concept for electron cyclotron emission imaging ${ }^{\text {a) }}$
}

\author{
J. X. Liu, ${ }^{1, b)}$ T. Milbourne,${ }^{2}$ M. Bitter, ${ }^{3}$ L. Delgado-Aparicio, ${ }^{3}$ A. Dominguez, ${ }^{3}$ \\ P. C. Efthimion, ${ }^{3}$ K. W. Hill, ${ }^{3}$ G. J. Kramer, ${ }^{3}$ C. Kung, ${ }^{3}$ S. Kubota,,${ }^{4}$ W. Kasparek, ${ }^{5}$ \\ J. Lu, ${ }^{6}$ N. A. Pablant, ${ }^{3}$ H. Park, ${ }^{7}$ and B. Tobias ${ }^{3}$ \\ ${ }^{1}$ Department of Physics, University of California Berkeley, Berkeley, California 94720, USA \\ ${ }^{2}$ Department of Physics, College of William and Mary, Williamsburg, Virginia 23185, USA \\ ${ }^{3}$ Princeton Plasma Physics Laboratory, Princeton, New Jersey 08540, USA \\ ${ }^{4}$ Department of Physics, University of California Los Angeles, Los Angeles, California 90095, USA \\ ${ }^{5}$ Department of Electrical Engineering, University of Stuttgart, Stuttgart, Germany \\ ${ }^{6}$ Department of Physics, Chongqing University, Chongqing 400044, China \\ ${ }^{7}$ Ulsan National Institute of Science and Technology, Ulsan 689-798, South Korea
}

(Presented 3 June 2014; received 31 May 2014; accepted 11 June 2014; published online 30 June 2014)

The implementation of advanced electron cyclotron emission imaging (ECEI) systems on tokamak experiments has revolutionized the diagnosis of magnetohydrodynamic (MHD) activities and improved our understanding of instabilities, which lead to disruptions. It is therefore desirable to have an ECEI system on the ITER tokamak. However, the large size of optical components in presently used ECEI systems have, up to now, precluded the implementation of an ECEI system on ITER. This paper describes a new optical ECEI concept that employs a single spherical mirror as the only optical component and exploits the astigmatism of such a mirror to produce an image with one-dimensional spatial resolution on the detector. Since this alternative approach would only require a thin slit as the viewing port to the plasma, it would make the implementation of an ECEI system on ITER feasible. The results obtained from proof-of-principle experiments with a $125 \mathrm{GHz}$ microwave system are presented. ( 2014 AIP Publishing LLC. [http://dx.doi.org/10.1063/1.4884902]

\section{INTRODUCTION}

The implementation of advanced electron cyclotron emission imaging (ECEI) systems on the major tokamak experiments, TEXTOR, ${ }^{1}$ DIII-D, ${ }^{2,3}{ }^{\mathrm{KSTAR}},{ }^{4} \mathrm{EAST}^{5},{ }^{6}$ and ASDEX Upgrade $^{6}$ has revolutionized the diagnosis of MHD activities and improved our understanding of instabilities, which lead to disruptions. ${ }^{7-9}$ It is therefore desirable to have an ECEI system on ITER. However, the large size optical components used on present ECEI systems, which include up to ten, $1 \mathrm{~m}$ high, cylindrical lenses and which require access ports of the size of a manhole, have precluded the implementation of an ECEI system on ITER. Here, we propose an alternative optical concept, which would make the implementation of an ECEI system on ITER feasible since a narrow, $1-3 \mathrm{~cm}$ wide, vertical slit in the ITER wall would suffice as the viewing port to the plasma. This alternative concept employs a single spherical mirror as the only optical component and exploits the astigmatism of such a mirror for two-dimensional imaging. Spatial resolution would be obtained perpendicular to the toroidal magnetic field. This imaging scheme is very scalable and can easily match the dimensions of a large tokamak such as ITER. This concept is already being used in the design of $\mathrm{x}$-ray imaging crystal spectrometers. ${ }^{10-13}$ However, an application to microwaves, whose wavelengths are not neg-

\footnotetext{
a) Contributed paper, published as part of the Proceedings of the 20th Topical Conference on High-Temperature Plasma Diagnostics, Atlanta, Georgia, USA, June 2014.

b) Author to whom correspondence should be addressed. Electronic mail: jsliu9@berkeley.edu
}

ligible against the dimensions of optical components, requires additional testing, since diffraction effects could cause image distortions. We have therefore performed proof-of-principle experiments with microwaves, which are described in this paper.

\section{ALTERNATIVE ECEI CONCEPT}

Figure 1 illustrates the imaging properties of a spherical reflector and shows the arrangement of a spherically bent crystal that is presently being used in one-dimensional x-ray imaging spectrometers on tokamak experiments for measurements of the ion-temperature and plasma-flow-velocity profiles. Due to the astigmatism of a spherical mirror, a point source, located at $P$, will not be imaged onto a point but rather into two mutually perpendicular, meridional and sagittal focal lines at $B m$ and Bs. Vice versa, rays emanating from or passing through the sagittal focal line at $B s$ in the plasma will be imaged onto the point $P$. Since the ray pattern is symmetric with respect to rotations about the spherical mirror's normal axis, $O C$, one obtains spatial resolution in a direction perpendicular to the toroidal magnetic field. By such an imagined rotation of the ray pattern, the sagittal focal line $B s$ and the point $P$ would move up or down in opposite directions on the opposing sites of a cone about $O C$. With our system, as with the current systems, ${ }^{3-7}$ spatial resolution along the major radius $\mathrm{R}$ of the plasma would be achieved by analyzing the frequency of the emitted ECE radiation, since the toroidal magnetic field (and thus the ECE frequency) varies inversely with the major radius. 


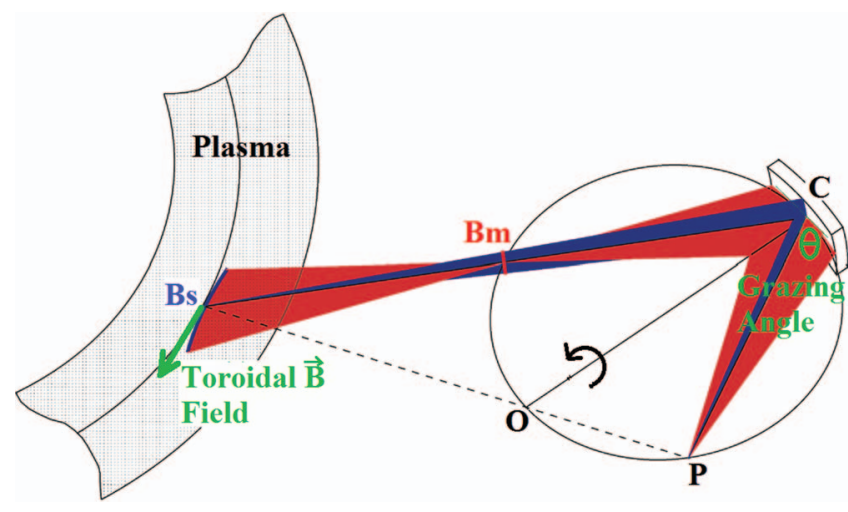

FIG. 1. The imaging properties of a spherical mirror and its typical arrangement on tokamaks for diagnostic applications.

\section{EXPERIMENTS}

The imaging properties of the scheme shown in Fig. 1 were tested with the following experimental parameters:

- Microwave frequency: $\mathrm{f}=125 \mathrm{GHz}$.

- Radius of curvature of spherical mirror: $\mathrm{R}=1 \mathrm{~m}$.

- Diameter of spherical mirror: $\mathrm{D}=15 \mathrm{~cm}$.

- Grazing angle: $\Theta=60^{\circ}$.

- Distance from point $\mathrm{P}$ to mirror: $C P=\mathrm{R} \sin (\Theta)$ $=86.6 \mathrm{~cm}$.

- Distance from meridional focal line to mirror: $\mathrm{CBm}$ $=\mathrm{R} \sin (\Theta)=86.6 \mathrm{~cm}$.

- Distance from sagittal focal line to mirror: $C B s$ $=-\mathrm{R} \sin (\Theta) / \cos (2 \Theta)=173.2 \mathrm{~cm}$.

A $125 \mathrm{GHz}$ Gunn oscillator was used as the microwave source and an ELVA-1 Power Meter was used as the detector. The transmitter and receiver antennae were horn antennae with apertures of $2.8 \times 2.1 \mathrm{~cm}^{2}$ and $2.1 \times 1.6 \mathrm{~cm}^{2}$, respectively. The spherical mirror was fabricated from solid aluminum.

\section{A. Reflected beam profiles}

The transmitter antenna was placed at $P$, and the horizontal, 1D intensity profiles of the reflected radiation were measured at different distances from the mirror. This experimental setup did not include a slit.

The profiles obtained are shown in Figure 2. The width of the intensity profiles varies with the distance from the mirror as expected from the ray pattern shown in Fig. 1. The narrowest and most peaked profile appears at a distance of $86.6 \mathrm{~cm}$, which corresponds to the position of the meridional focus. Effects due to diffraction or interference of the incident and reflected radiation were also observed. Those effects are most prominent in the intensity profiles in the vicinity of the mirror and less prominent in the intensity profiles farther away from the mirror.

Figure 3 shows a two-dimensional intensity profile measured at the position of the sagittal focus. The $-20 \mathrm{~dB}$ width of the profile in the horizontal (x) direction is about $16 \mathrm{~cm}$, and the FWHM is approximately $4 \mathrm{~cm}$. In the vertical (z) direction, focusing is much tighter, with a $-20 \mathrm{~dB}$ width of $8 \mathrm{~cm}$ and a FWHM of $2.5 \mathrm{~cm}$.
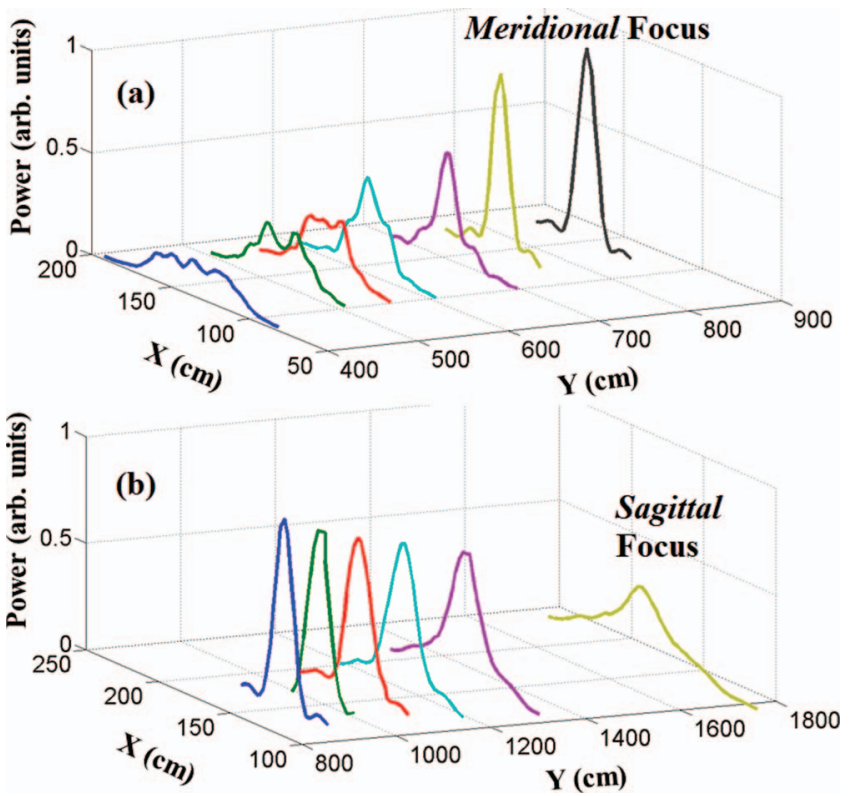

FIG. 2. (a) One-dimensional intensity profiles in the reflected beam, measured in the $\mathrm{x}$-direction at the distances $\mathrm{y}=40.6,50.6,55.6,60.6,69.6,75.6$, and $86.6 \mathrm{~cm}$ from the mirror. (b) One-dimensional intensity profiles in the reflected beam, measured in the x-direction at the distances 91.6, 98.6, 108.6, $118.6,133.6$, and $173.6 \mathrm{~cm}$ from the mirror.

The widths of the profile in the vertical and horizontal dimensions are consistent with theoretical expectations. The imaging system has a magnification of $\mathrm{M}=2$ for the grazing angle of $\Theta=60^{\circ}$. Therefore, the vertical spot size is expected to be about twice the transmitter horn antenna's vertical waist size of $1.3 \mathrm{~cm}$ and be unaffected by slit diffraction. The width of the profile in the horizontal dimension should be equal to the width of the mirror, and will become wider due to slit diffraction.

\section{B. System reciprocity}

To access the reciprocity of the system, the microwave source was placed at various positions along the sagittal focal line, at the distance $\mathrm{y}=173.1 \mathrm{~cm}$ from the mirror, and intensity profiles were measured near the point $P$ along the horizontal dimension. In this experimental arrangement, a vertical slit of variable width was introduced at the meridional focus. The experimental arrangement included a vertical slit of variable width at the meridional focus.

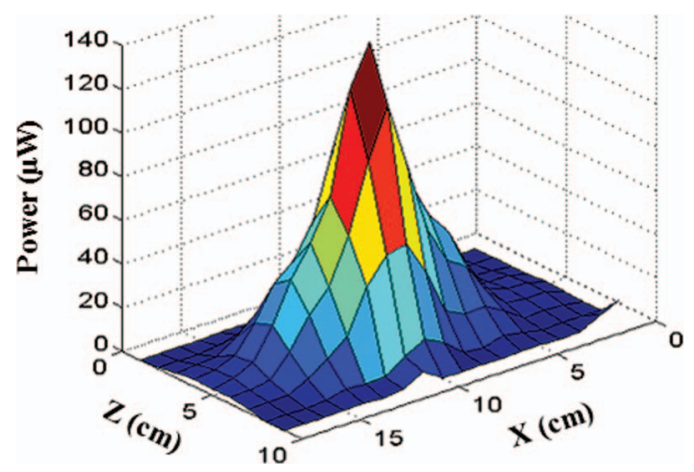

FIG. 3. Two-dimensional intensity profile measured at the position of the sagittal focus, at a distance of $173.1 \mathrm{~cm}$. 

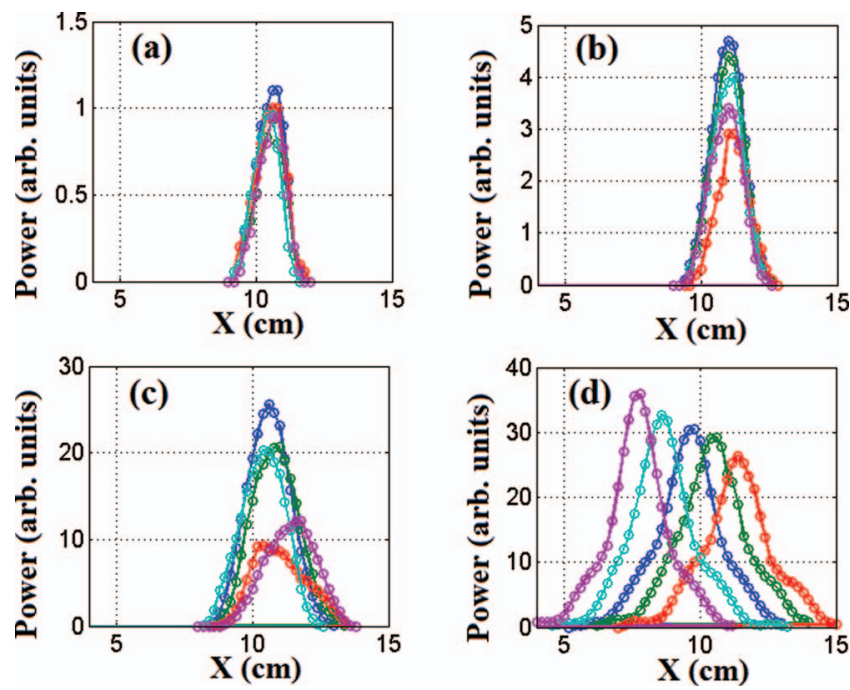

FIG. 4. Intensity profiles measured at $P$ for different source positions along the sagittal focus, using a slit of width (a) $0.5 \mathrm{~cm}$, (b) $1.0 \mathrm{~cm}$, (c) $3.0 \mathrm{~cm}$, and (d) no slit.

Figure 4 shows the intensity profiles obtained for the source positions $\mathrm{x}=-4,-3,-2,-1,0,1,2,3$, and $4 \mathrm{~cm}$ measured from the center of the sagittal focal line along the sagittal focal line. For slit widths of 0.5 and $1.0 \mathrm{~cm}$, the peak position of the intensity profiles did not depend on the source position along the sagittal focus, as seen in Figs. 4(a) and 4(b). This is due to the fact that, with a sufficiently narrow slit, the radiation emitted from different source positions strikes different parts of the mirror with the same grazing angle $\Theta$. The reflected radiation is therefore focused to the same point, $\mathrm{P}$. On the other hand, if the slit is removed, each source point irradiates the entire mirror. In this case, the grazing angle $\Theta$ and therefore the peak positions of the profiles observed near $P$ (see Fig. 4(d)) vary as expected from the imaging equations for a spherical mirror. The profiles obtained with a slit width of $3 \mathrm{~cm}$, as seen in Fig. 4(c), represent an intermediate case, since the peak positions of the profiles obtained for the outermost source positions of -4 and $4 \mathrm{~cm}$ are offset.

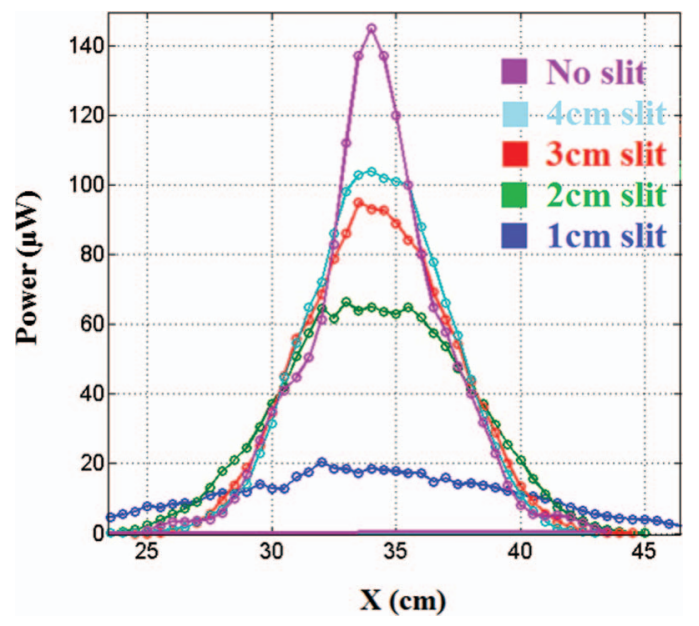

FIG. 5. Intensity profiles measured at the sagittal focus, $B s$, for a slit with variable slit width placed at the meridional focus, $\mathrm{Bm}$.

\section{Diffraction}

To analyze the slit's diffractive effects, a vertical slit of variable width was placed at the meridional focus and intensity profiles were measured for different slit widths along the sagittal focal line (x-direction), which was perpendicular to the slit. The microwave source was positioned at $P$. The experimental results obtained are shown in Fig. 5.

As expected, diffraction effects caused a broadening of the intensity profile and became more prominent with decreasing slit widths. However, this profile broadening occurs along the sagittal focal line, which would be aligned parallel to the toroidal magnetic field in a tokamak experiment. It would not impair spatial resolution in the direction of interest, perpendicular to the toroidal magnetic field, because that direction is parallel to the slit and is unaffected by diffraction.

\section{CONCLUSIONS}

Our experimental results demonstrate that the ray pattern required for our alternative ECEI concept can be established with microwaves. This concept could therefore be employed for the implementation of an ECEI system on ITER that would match the diagnostic capabilities of the advanced ECEI systems on DIII-D, EAST, and KSTAR. The advantages of this alternative concept for ITER are obvious: a single spherical mirror would be the only optical component and a narrow 2-3 cm wide slit in the ITER wall would provide viewing access to a significant portion of the ITER plasma. The neutron and gamma radiation from the plasma would thereby be substantially reduced, lowering the risks of radiation damage and signal distortion of the ECEI diagnostic. The ECE detector antennae would be arranged in a vertical array to simultaneously record the ECE radiation from different vertical locations in the plasma. With two vertical rows of detectors employing two vertical slits and two different grazing angles on the same spherical mirror, it would be possible to simultaneously record the ECE signals from two different toroidal locations and perform cross-correlation measurements. Further tests of our alternative ECEI system, employing the KSTAR tokamak's ECEI detector arrays, are currently being planned.

\section{ACKNOWLEDGMENTS}

We gratefully acknowledge the support of this work by the US Department of Energy Contract No. DE-AC02-09CH11466 and Korean NRF Contract No. 20120005920.

${ }^{1}$ H. Park, E. Mazzucato, T. Munsat et al., Rev. Sci. Instrum. 75, 3787 (2004). ${ }^{2}$ B. Tobias, C. Domier, and T. Liang, Rev. Sci. Instrum. 81, 10 D928 (2010).

${ }^{3}$ T. Liang, B. Tobias, and X. Kong, Rev. Sci. Instrum. 81, 10D909 (2010).

${ }^{4}$ G. S. Yun, W. Lee, and M. J. Choi, Rev. Sci. Instrum. 81, 10D930 (2010).

${ }^{5} \mathrm{C}$. W. Domier et al., "Electron cyclotron emission diagnostics for EAST," in paper presented at the 19th High-Temperature Plasma Diagnostics Topical Conference in Monterey, CA, 6-10 May 2012, P.2.22.

${ }^{6}$ I. G. J. Classen et al., Rev. Sci. Instrum. 81, 10D929 (2010).

${ }^{7}$ G. S. Yun, W. Lee, and M. J. Choi, Phys. Rev. Lett. 107, 045004 (2011).

${ }^{8}$ B. J. Tobias et al., Phys. Rev. Lett. 106, 075003 (2011).

${ }^{9}$ T. Munsat, C. W. Domier, and X. Kong, Appl. Opt. 49, E20-E30 (2010).

${ }^{10}$ A. Ince-Cushman et al., Rev. Sci. Instrum. 79, 10E302 (2008).

${ }^{11}$ M. Bitter et al., J. Phys. B: At. Mol. Opt. Phys. 43, 144011 (2010).

${ }^{12}$ S. G. Lee et al., Rev. Sci. Instrum. 81, $10 \mathrm{E} 506$ (2010).

${ }^{13}$ N. A. Pablant et al., Rev. Sci. Instrum. 83, 083506 (2012). 